\title{
Salud mental infanto-juvenil en Chile y brechas de atención sanitarias
}

\author{
BENJAMÍN VICENTE ${ }^{1}$, SANDRA SALDIVIA ${ }^{1}$, \\ FLORA DE LA BARRA ${ }^{2}$, ROBERTO MELIPILLÁN ${ }^{1}$, \\ MARIO VALDIVIA ${ }^{1}$, ROBERT KOHN ${ }^{3}$
}

\section{Prevalence of psychiatric disorders among Chilean children and adolescents}

Background: Psychiatric disorders that appear during childhood or adolescence should be a public health priority. Aim: To report the results of a national representative survey in the Latin American region examinig the prevalence of DSM-IV psychiatric disorders in children and adolescents. Material and Methods: Subjects aged 4 to 18 years were selected using a stratified multistage design. The Diagnostic Interview Schedule for Children Version IV (DISC-IV) was used to obtain 12-month $D S M-I V$ diagnoses, and was supplemented with questionnaires examining family risk factors, socioeconomic status and service use. Results: A sample of 1558 children and adolescents (51\% males) was evaluated. Fifty three percent of the sample were children aged 4 to 11 years and the rest were adolescents aged 12 to 18 years. The prevalence rate for any psychiatric disorder without impairment was 38.3\% (33.5\% for boys and $43.3 \%$ for girls). The prevalence rate was higher among children as compared to adolescents (42.9\% and 33.2\%, respectively). A third of participants receiving a diagnosis sought some form of assistance. Nearly a quarter of those using services, did not have a psychiatric diagnosis in the past year. Comorbidity was found in $27 \%$ of those with a disorder, but only $7 \%$ had three or more diagnoses. Conclusions: The prevalence of psychiatric disorders in Chile is high among children and adolescents. This study highlights the increasing need to re-evaluate mental health services provided to children and adolescents in our country.

(Rev Med Chile 2012; 140: 447-457).

Key words: Adolescent; Chile; Epidemiology; Mental disorders, prevalence.
'Departmento de Psiquiatría y Salud Mental, Universidad de Concepción

Concepción, Chile. 2Escuela de Medicina, Universidad de Chile, Santiago, Chile. ${ }^{3}$ Departament of Psychiatry and Human Behavior, Warren Alpert Medical School of Brown University, Providence, RI, USA.

Estudio financiado por el Proyecto FONDECYT No1070519.

Recibido el 14 de septiembre de 2011 aceptado el 20 de diciembre de 2011

Correspondencia a: Benjamin Vicente, MD, PhD; Casilla 160-C, Departamento de Psiquiatría y Salud Mental, Universidad de Concepción Concepción, Chile. Tel-fax: 56-41-2312799 E-mail: bvicent@udec.cl
L a Organización Mundial de la Salud (OMS) ha establecido que los trastornos psiquiátricos que se inician en la infancia o adolescencia deben ser una prioridad para la Salud Pública. El estudio norteamericano ECA de población adulta, en un análisis retrospectivo, demuestra que la edad de inicio de un número importante de trastornos ocurre antes de lo que se estimaba ${ }^{1}$. Recientemente el estudio NCS-R, también en Estados Unidos de Norteamérica confirmó el hallazgo².

Estudios prospectivos de cohortes de niños nacidos en la década 1960-69 identificaron claramente dos grupos de trastornos, aquellos que se inician en la infancia y en la adolescencia ${ }^{3,4}$. Sin embargo, datos sobre la prevalencia de estos trastornos son aún insuficientes especialmente en países en desarrollo ${ }^{5,6}$.

A pesar de la eficacia demostrada por muchas de las intervenciones terapéuticas disponibles para los trastornos psiquiátricos de la infancia y adolescencia ${ }^{7}$ y de la evidencia que apoya la importancia de medidas de prevención y de promoción de ambientes saludables ${ }^{8}$, muchos niños $\mathrm{y}$ adolescentes no reciben los servicios necesarios. En Estados Unidos de Norteamérica un adulto con depresión tiene 14 veces más posibilidades de reci- 
bir atención y $89 \%$ de los gastos en Salud Mental se destinan a población adulta9 ${ }^{9}$ La OMS enfatiza que el primer paso en la planificación de servicios de Salud Mental es la recolección de información relevante. El proyecto Atlas de recursos en Salud Mental fue diseñado con tal fin y confirma la escasa información disponible, en especial la brecha que existe entre la información sobre adultos y sobre la población infanto juvenil. La información disponible en Sudamérica sobre prevalencia y uso de servicios en este grupo etáreo es muy escasa ${ }^{10}$.

El presente trabajo es el primero realizado en un país hispanoparlante, al sur de México, que utiliza instrumentos diagnósticos estandarizados, e incorpora niños mayores de cuatro años ${ }^{11}$.

En Chile se disponía de estudios realizados en población escolarizada ${ }^{12}$ con la obvia limitación de quizá no representar toda la población más vulnerable ya que aun cuando es obligatorio algunos pudieron no estar asistiendo a la escuela.

El propósito de este estudio es determinar la prevalencia de trastornos psiquiátricos en una muestra representativa de la población infanto juvenil de Chile y las brechas de atención sanitarias.

\section{Material y Método}

El estudio fue conducido por el Departamento de Psiquiatría y Salud Mental de la Universidad de Concepción y el Departamento de Salud Mental Oriente de la Universidad de Chile, entre abril de 2007 y diciembre de 2009.

\section{Muestra}

Se diseñó una muestra aleatoria probabilística de la población infanto juvenil chilena entre los 4 y 18 años. Los casos se obtuvieron de cuatro provincias, Santiago, Concepción, Iquique y Cautín, consideradas representativas de la distribución nacional de la población, diseño ya utilizado en un estudio de población adulta ${ }^{13}$.

Comunas y manzanas fueron aleatoriamente seleccionadas. El número de viviendas requeridas por manzana se determinó usando el Censo del año 2002.

El tamaño muestral se definió considerando una prevalencia estimada de $18 \%$ de trastornos psiquiátricos basada en el estudio de Puerto Rico que también uso el DISC-IV ${ }^{14}$, con un intervalo de confianza de $95 \%$ y un error máximo estándar de $1,75 \%$.

\section{Instrumentos}

La presencia de un trastorno psiquiátrico fue evaluada usando la versión computarizada en español de DISC-IV ${ }^{15}$, validado en Chile por el equipo investigador ${ }^{16}$.

Las entrevistas se realizaron en el domicilio, directamente a los adolescentes entre 12 y 18 años o a los padres o cuidadores de los niños entre 4 y 11 años, por estudiantes de los últimos cursos de las carreras de psicología previamente entrenados en el uso de los instrumentos.

El DISC-IV permite además determinar el nivel de discapacidad asociada, la que puede clasificarse en cuatro criterios que van desde $A$ hasta $D$, siendo inclusivos y de menor a mayor exigencia. El criterio de discapacidad $\mathrm{D}$, es definido como la presencia de discapacidad en un ámbito del quehacer diario considerada grave (auto reportada), evaluada de acuerdo con la intensidad con que los síntomas afectan el funcionamiento personal y escolar de los jóvenes o su relación con los cuidadores, familiares o profesores, o la interferencia en dos ámbitos con una valoración de intensidad intermedia.

Se presentan y discuten las prevalencias sin impedimento, considerando que su valor como medida única de discapacidad ha sido cuestionada sugiriendo que los adolescentes la minimizan y los padres y cuidadores la exageran ${ }^{15}$.

Se investigó además la utilización de servicios, tanto públicos como privados en los últimos 12 meses, por problemas emocionales, conductuales $\mathrm{y}$ asociados a consumo de sustancias.

Se definieron como servicios educacionales a la consejería, educación especial u otra intervención educacional para problemas conductuales o de aprendizaje. Servicios de salud mental formales incluyeron tratamiento psiquiátrico ambulatorio u hospitalizado y atención por otros profesionales de salud mental. Otros servicios de salud incluyeron cualquier atención sanitaria no entregada por profesional de salud mental. Servicios sociales incorporaron cualquier atención psico-social que no ocurriera en el ámbito sanitario o educacional tales como programas del SENAME, Centros de Detención, etc. Otros servicios considera el apoyo de líderes religiosos, curanderos u otros grupos sociales.

La información sobre el uso de servicio de salud mental se recogió con una versión modificada del Service Assesment for Children and Adolescent $(\mathrm{SACA})^{17}$. 
El funcionamiento familiar se evaluó usando la escala de 12 ítems del Family Assesment Device (FAD), sus reactivos recogen información de forma tal que al procesarlos, los puntajes más altos representan un mejor o más adecuado funcionamiento familiar ${ }^{18,19}$.

La historia psiquiátrica familiar se obtuvo usando el Family History Screen (FHS) ${ }^{20}$. El nivel socioeconómico se determinó considerando el ingreso familiar global sobre la línea de pobreza definida en Chile a noviembre de 2009 de US\$ 4.249,46 dólares por año para áreas rurales y US\$ 6.334,32 para áreas urbanas.

Las entrevistas fueron todas revisadas por los investigadores y las discrepancias resueltas con los entrevistadores lo que ocasionalmente significó visitar nuevamente al entrevistado.

Con la finalidad de no alterar el diseño muestreal, no fueron reemplazadas aquellas viviendas donde se rehusó participar, sólo aquellas donde no vivían niños o adolescentes. Las principales razones para el rechazo fueron "falta de tiempo" y "de interés"; la post-estatificación final de la muestra por sexo y edad corrige eventuales distorsiones asociadas a los rechazos.

\section{Análisis estadístico}

La información complementaria no recogida en el DISC-IV fue ingresada con doble digitación para minimizar errores, en una base de datos SPSS.

El DISC-IV entrega diagnósticos DSM-IV presentes en los últimos 12 meses. Las prevalencias estimadas con sus errores standares se obtuvieron usando el programa STATA 11.0 que considera diseños muestrales complejos. Se usó el análisis de regresión logística multivariada para determinar la asociación de los trastornos mentales con variables sociodemográficas y otros potenciales factores de riesgo. El método de linearización de Taylor fue empleado para calcular los errores estándar, intervalos de confianza y valores $\mathrm{p}$.

La regresión logística se usó para determinar cuáles factores de riesgo predecían mejor los trastornos mentales. Las variables que se incorporaron al modelo de regresión logística multivariada se seleccionaron en base al estadístico F de Rao Scott con una significación estadística menor a $0,25^{21}$.

La muestra fue pesada para ajustar la probabilidad de selección en cada etapa: comuna, manzana, vivienda y niño o adolescente. Adicionalmente se realizó una post-estratificación para ajustar la muestra según sexo y edad al Censo 2002.

Se obtuvo un consentimiento informado de los adultos responsables de cada niño y de los adolescentes que participaron en el estudio. Toda vez que fue solicitado, la información sobre diagnósticos se entregó a los responsables sin que esto asegurara una atención oportuna. Los Comités de Ética de la Universidad de Concepción y FONDECYT aprobaron el estudio.

\section{Resultados}

Un total de 1.558 sujetos fueron entrevistados, $50,9 \%$ hombres y 49,1\% mujeres. Más de la mitad de la muestra, 52,9\%, fueron niños entre 4-11 años y $47,1 \%$ adolescentes entre 12 y 18 años. La tasa de respuesta alcanzó al 82,4\%.

La Tabla 1 muestra las prevalencias de los diagnósticos estudiados. La prevalencia global general alcanzó a 38,3\% que disminuyó a 22,5\% cuando se considera el impedimento D. El grupo diagnóstico más prevalente fueron los trastornos disruptivos con 21,8\%; seguido por los trastornos ansiosos, $18,5 \%$; y los afectivos con $6,1 \%$. Los trastornos asociados al consumo de sustancias alcanzaron a 4,8\%; la esquizofrenia y los trastornos alimentarios fueron comparativamente escasos.

\section{Análisis bivariado}

La Tabla 2 muestra la relación entre los grupos diagnósticos y las principales características socio demográficas. Las mujeres tenían un riesgo mayor de hacer trastornos ansiosos $(\mathrm{OR}=1,9,95 \% \mathrm{CI}$ $[1,3-2,8])$ y afectivos (OR $=2,5,95 \%$ CI $[1,2-4,9])$, lo que se repite o confirma cuando se considera la prevalencia global o cualquier trastorno estudiado $(\mathrm{OR}=1,5,95 \%$ CI $[1,1-2,2])$.

Los adolescentes tuvieron más probabilidad de presentar cuadros afectivos $(\mathrm{OR}=2,4,95 \% \mathrm{CI}$ $[1,2-4,6])$ y claramente menos cuadros disruptivos y cualquiera de los trastornos estudiados. Un nivel socioeconómico alto aparece significativamente asociado a la menor probabilidad de presentar trastornos ansiosos.

La percepción de disfunción familiar estuvo significativamente asociada con la presencia de todos los trastornos estudiados al igual que el antecedente de psicopatología en la familia, con la excepción de los trastornos asociados al consumo de sustancias. 
Tabla 1. Prevalencia de trastornos psiquiátricos (DSM-IV) en el último año sin impedimento en población infanto-juvenil $(n=1.558)$

\begin{tabular}{|c|c|c|c|c|c|c|c|c|c|c|}
\hline & To & & Varc & nes & Muj & res & 4-11 & años & $12-18$ & años \\
\hline & & EE & & EE & & EE & & EE & & EE \\
\hline Trastornos ansiosos & 18,5 & 1,1 & 13,8 & 1,4 & 23,5 & 2,1 & 21,0 & 1,8 & 15,7 & 1,9 \\
\hline Fobia social & 5,5 & 0,9 & 2,9 & 0,8 & 8,2 & 1,6 & 5,9 & 1,2 & 5,1 & 1,3 \\
\hline Trastorno de ansiedad generalizada & 8,2 & 0,9 & 6,2 & 1,3 & 10,3 & 1,3 & 8,0 & 1,7 & 8,4 & 1,6 \\
\hline Trastorno por ansiedad de separación & 10,2 & 8,8 & 7,3 & 0,9 & 13,1 & 1,3 & 13,4 & 1,2 & 6,5 & 1,5 \\
\hline Trastornos afectivos & 6,1 & 0,8 & 3,7 & 1,0 & 8,6 & 1,4 & 3,8 & 1,1 & 8,6 & 1,2 \\
\hline Trastorno depresivo mayor & 5,9 & 0,8 & 3,6 & 1,0 & 8,4 & 1,4 & 3,8 & 1,1 & 8,3 & 1,1 \\
\hline Distimia & 0,2 & 0,1 & 0,1 & 0,1 & 0,2 & 0,2 & 0,04 & 0,03 & 0,3 & 0,2 \\
\hline Trastornos por comportamiento disruptivo & 21,8 & 1,8 & 19,9 & 2,1 & 23,7 & 2,8 & 29,7 & 3,7 & 12,8 & 1,4 \\
\hline Trastorno disocial & 3,7 & 0,5 & 4,4 & 0,9 & 3,0 & 0,7 & 1,2 & 0,3 & 6,5 & 1,0 \\
\hline Trastornos oposicionista desafiante & 8,7 & 1,0 & 7,5 & 1,1 & 9,9 & 1,4 & 11,8 & 1,5 & 5,1 & 1,2 \\
\hline TDAH & 15,1 & 1,2 & 13,7 & 1,6 & 16,6 & 2,2 & 23,9 & 2,2 & 5,4 & 1,4 \\
\hline Trastornos por consumo de sustancias & 4,8 & 0,8 & 4,6 & 1,0 & 4,9 & 1,0 & - & - & 10,1 & 1,7 \\
\hline Abuso de alcohol & 1,9 & 0,6 & 2,4 & 0,8 & 1,3 & 0,8 & - & - & 4,0 & 1,3 \\
\hline Dependencia de alcohol & 1,7 & 0,4 & 1,5 & 0,5 & 1,9 & 0,7 & - & - & 3,6 & 0,9 \\
\hline Abuso de marihuana & 0,4 & 0,2 & 0,2 & 0,1 & 0,6 & 0,4 & - & - & 0,9 & 0,3 \\
\hline Dependencia de marihuana & 0,4 & 0,2 & 0,3 & 0,2 & 0,5 & 0,2 & - & - & 0,9 & 0,3 \\
\hline Dependencia de nicotina & 1,6 & 0,4 & 1,4 & 0,5 & 1,8 & 0,6 & - & - & 3,4 & 0,8 \\
\hline Abuso de otras sustancias & 0,2 & 0,1 & 0,2 & 0,2 & 0,2 & 0,2 & - & - & 0,4 & 0,2 \\
\hline Dependencia de otras sustancias & 0,2 & 0,1 & 0,2 & 0,2 & 0,2 & 0,2 & - & - & 0,4 & 0,2 \\
\hline Trastorno alimenticio & 0,3 & 0,2 & 0,05 & 0,05 & 0,5 & 0,3 & 0,2 & 0,2 & 0,3 & 0,3 \\
\hline Esquizofrenia & 0,2 & 0,2 & 0,2 & 0,2 & 0,3 & 0,3 & 0,2 & 0,2 & 0,3 & 0,3 \\
\hline Cualquier trastorno & 38,3 & 2,3 & 33,5 & 2,5 & 43,3 & 3,6 & 42,9 & 3,6 & 33,2 & 2,4 \\
\hline
\end{tabular}

Datos Pesados Censo Chile 2002.

Vivir solo con un padre fue fuertemente asociado con la presencia de trastornos psiquiátricos y deserción escolar se asoció sólo con la presencia de trastornos por uso de sustancias $(\mathrm{OR}=6,8$, $95 \%$, CI $[3,4-13,8])$.

\section{Regresión logística múltiple}

Los resultados del modelo del análisis de regresión logística múltiple se presentan en la Tabla 3. Para los trastornos ansiosos, la asociación con el sexo, la pobreza, la historia de psicopatología en la familia y vivir con un solo padre se mantuvo significativa. Condición que se restringe a sexo, psicopatología familiar y no vivir con ambos padres en los trastornos afectivos. La edad y el funcionamiento familiar fueron significativos para los trastornos disruptivos y deserción escolar se mantiene para los trastornos por uso de sustancias.

\section{Comorbilidad}

La comorbilidad, sin considerar impedimentos $(n=560)$, estuvo presente en un cuarto $(26,6 \%)$ de los que tienen un trastornos y 7,6\% de estos tenían 3 o más diagnósticos. 
Salud mental infanto-juvenil en Chile - B. Vicente et al

Tabla 2. Correlaciones socio-demográficas de los trastornos psiquiátricos estudiados, sin impedimento ( $n=1.558)$

\begin{tabular}{|c|c|c|c|c|c|c|c|c|c|c|}
\hline & \multicolumn{2}{|c|}{$\begin{array}{c}\text { Trastornos } \\
\text { ansiosos }\end{array}$} & \multicolumn{2}{|c|}{$\begin{array}{l}\text { Trastornos } \\
\text { afectivos }\end{array}$} & \multicolumn{2}{|c|}{$\begin{array}{l}\text { Trastornos por } \\
\text { comportamiento } \\
\text { disruptivo }\end{array}$} & \multicolumn{2}{|c|}{$\begin{array}{l}\text { Trastornos por } \\
\text { consumo de alco- } \\
\text { hol y sustancias }\end{array}$} & \multicolumn{2}{|c|}{$\begin{array}{l}\text { Cualquier } \\
\text { trastorno }\end{array}$} \\
\hline & OR & IC $95 \%$ & OR & IC $95 \%$ & OR & IC 95\% & OR & IC $95 \%$ & OR & IC $95 \%$ \\
\hline \multicolumn{11}{|l|}{ Género } \\
\hline Masculino & 1,0 & & 1,0 & & 1,0 & & 1,0 & & 1,0 & \\
\hline Femenino & $1,9 * * *$ & $(1,3-2,8)$ & $2,5^{*}$ & $(1,2-4,9)$ & 1,2 & $(0,9-1,8)$ & 1,1 & $(0,7-1,6)$ & $1,5^{*}$ & $(1,1-2,2)$ \\
\hline \multicolumn{11}{|l|}{ Edad } \\
\hline $4-11$ & 1,0 & & 1,0 & & 1,0 & & - & - & 1,0 & \\
\hline $12-18$ & 0,7 & $(0,5-1,1)$ & $2,4^{*}$ & $(1,2-4,6)$ & $0,4^{* * *}$ & $(0,2-0,6)$ & - & - & $0,7^{*}$ & $(0,5-0,9)$ \\
\hline \multicolumn{11}{|l|}{ NSE } \\
\hline Pobreza $\leq 2$ & 1,0 & & 1,0 & & 1,0 & & 1,0 & & 1,0 & \\
\hline Pobreza $\leq 5$ & 0,9 & $(0,5-1,5)$ & 0,9 & $(0,5-1,6)$ & 1,8 & $(0,9-3,4)$ & 0,8 & $(0,3-2,1)$ & 1,4 & $(0,8-2,3)$ \\
\hline Pobreza $\leq 8$ & 0,6 & $(0,3-1,3)$ & 0,6 & $(0,2-1,3)$ & 1,6 & $(0,7-3,5)$ & 0,7 & $(0,2-2,1)$ & 1,1 & $(0,6-2,3)$ \\
\hline Pobreza $>8$ & $0,5^{*}$ & $(0,3-0,9)$ & 0,6 & $(0,2-1,5)$ & 1,3 & $(0,5-3,2)$ & 1,6 & $(0,6-4,5)$ & 1,3 & $(0,7-2,6)$ \\
\hline $\begin{array}{l}\text { Funcionamiento } \\
\text { familiar }\end{array}$ & $0,6^{* *}$ & $(0,4-0,9)$ & $0,3^{* *}$ & $(0,1-0,6)$ & $0,5^{* *}$ & $(0,3-0,8)$ & $0,4^{* *}$ & $(0,2-0,7)$ & $0,5^{* * *}$ & $(0,4-0,7)$ \\
\hline \multicolumn{11}{|c|}{ Psicopatología familiar } \\
\hline No & 1,0 & & 1,0 & & 1,0 & & 1,0 & & 1,0 & \\
\hline Sí & $1,9 * *$ & $(1,3-2,9)$ & $4,0 * * *$ & $(1,8-9,0)$ & $2,9^{* * *}$ & $(1,7-4,8)$ & 2,3 & $(1,0-5,4)$ & $2,4^{* * *}$ & $(1,8-3,2)$ \\
\hline \multicolumn{11}{|c|}{ Estructura familiar } \\
\hline Ambos padres & 1,0 & & 1,00 & & 1,0 & & 1,0 & & 1,0 & \\
\hline Sólo un padre & $2,0 * * *$ & $(1,5-2,7)$ & $3,0^{* * *}$ & $(1,8-5,0)$ & $1,9 * * *$ & $(1,4-2,6)$ & $1,9^{*}$ & $(1,1-3,6)$ & $2,1 * * *$ & $(1,5-2,9)$ \\
\hline Otro & $2,0^{*}$ & $(1,1-3,8)$ & $3,9 * * *$ & $(2,0-7,6)$ & 1,5 & $(0,9-2,5)$ & 2,5 & $(0,7-8,6)$ & $1,7^{*}$ & $(1,1-2,5)$ \\
\hline \multicolumn{11}{|l|}{ Deserción escolar } \\
\hline No & 1,0 & & 1,0 & & 1,0 & & 1,0 & & 1,0 & \\
\hline Sí & 1,3 & $(0,5-3,3)$ & 1,8 & $(0,9-3,7)$ & 0,6 & $(0,3-1,3)$ & $6,8^{* * *}$ & $(3,4-13,8)$ & 1,3 & $(0,7-2,3)$ \\
\hline
\end{tabular}

Las mujeres presentaron una mayor tendencia a presentar comorbilidad (Tabla 4). Un funcionamiento familiar pobre, la presencia de psicopatología en la familia y no vivir con ambos padres, representaron un mayor riesgo de presentar un trastornos psiquiátrico, independiente de la presencia de comorbilidad. Por otro lado, el efecto del nivel socioeconómico sólo se apreció en la comorbilidad de 3 o más trastornos y en el grupo de más altos ingresos, que aparece con una tendencia menor a presentar patologías comparados con los de ingresos más bajos.

\section{Utilización de servicios}

El 33,3\% de los sujetos entrevistados que presentan un trastorno habían buscado en el año previo ayuda formal o informal por problemas conductuales, emocionales, psiquiátricos o asociados a consumo de sustancias. La fuente más frecuente de ayuda fueron los servicios educacionales, $18,1 \%$; seguido de servicios formales de salud mental, $14,5 \%$; sólo $3,6 \%$ contactó a curanderos o medicinas alternativas. Especialmente destacable es la bajísima proporción en que se recurrió a servicios sociales considerando 
Tabla 3. Regresión logística multivariada de los trastornos psiquiátricos estudiados, sin impedimento ( $n=1.558)$

\begin{tabular}{|c|c|c|c|c|c|c|c|c|c|c|}
\hline & \multicolumn{2}{|c|}{$\begin{array}{l}\text { Trastornos } \\
\text { ansiosos }\end{array}$} & \multicolumn{2}{|c|}{$\begin{array}{l}\text { Trastornos } \\
\text { afectivos }\end{array}$} & \multicolumn{2}{|c|}{$\begin{array}{l}\text { Trastornos } \\
\text { por compor- } \\
\text { tamiento } \\
\text { disruptivo }\end{array}$} & \multicolumn{2}{|c|}{$\begin{array}{l}\text { Trastornos } \\
\text { por consumo } \\
\text { de alcohol y } \\
\text { sustancias }\end{array}$} & \multicolumn{2}{|c|}{$\begin{array}{l}\text { Cualquier } \\
\text { trastorno }\end{array}$} \\
\hline & OR & $95 \mathrm{Cl}$ & OR & $95 \mathrm{Cl}$ & OR & $95 \mathrm{Cl}$ & OR & $95 \mathrm{Cl}$ & OR & $95 \mathrm{Cl}$ \\
\hline \multicolumn{11}{|l|}{ Género } \\
\hline Masculino & 1,0 & & 1,0 & & 1,0 & & & & 1,0 & \\
\hline Femenino & $1,8^{* *}$ & $(1,2-2,8)$ & $2,6^{*}$ & $(1,2-6,0)$ & 1,2 & $(0,8-1,9)$ & & & $1,5^{*}$ & $(1,1-2,1)$ \\
\hline \multicolumn{11}{|l|}{ Edad } \\
\hline $4-11$ & 1,0 & & 1,0 & & 1,0 & & & & 1,0 & \\
\hline $12-18$ & 0,7 & $(0,4-1,1)$ & 2,1 & $(1,0-4,8)$ & $0,3^{* * *}$ & $(0,2-0,6)$ & & & $0,6^{*}$ & $(0,4-0,9)$ \\
\hline \multicolumn{11}{|l|}{ NSE } \\
\hline Pobreza $\leq 2$ & 1,0 & & & & & & 1,0 & & & \\
\hline Pobreza $\leq 5$ & 0,8 & $(0,5-1,4)$ & & & & & 0,8 & $(0,3-2,2)$ & & \\
\hline Pobreza $\leq 8$ & 0,6 & $(0,3-1,2)$ & & & & & 0,8 & $(0,3-2,3)$ & & \\
\hline Pobreza > 8 & $0,5^{*}$ & $(0,3-0,9)$ & & & & & 1,9 & $(0,7-5,4)$ & & \\
\hline $\begin{array}{l}\text { Funcionamiento } \\
\text { familiar }\end{array}$ & 0,7 & $(0,5-1,0)$ & 0,4 & $(0,1-1,1)$ & $0,5^{*}$ & $(0,2-0,8)$ & 0,7 & $(0,3-1,3)$ & $0,6^{* * *}$ & $(0,4-0,8)$ \\
\hline \multicolumn{11}{|c|}{ Psicopatología familiar } \\
\hline No & 1,0 & & 1,0 & & 1,0 & & 1,0 & & 1,0 & \\
\hline Sí & $1,9^{*}$ & $(1,2-3,0)$ & $3,3^{*}$ & $(1,5-7,6)$ & $2,5^{* *}$ & $(1,4-4,5)$ & 2,4 & $(1,0-5,9)$ & $2,1^{* * *}$ & $(1,5-2,8)$ \\
\hline \multicolumn{11}{|c|}{ Estructura familiar } \\
\hline Ambos padres & 1,0 & & 1,0 & & 1,0 & & 1,0 & & 1,0 & \\
\hline Sólo un padre & $1,8^{* *}$ & $(1,2-2,5)$ & $2,0^{*}$ & $(1,1-4,0)$ & $1,5^{*}$ & $(1,1-2,3)$ & 1,9 & $(1,0-3,6)$ & $1,9^{* * *}$ & $(1,3-2,7)$ \\
\hline Otro & 1,6 & $(0,9-3,1)$ & $2,8^{*}$ & $(1,2-6,4)$ & 1,0 & $(0,6-1,8)$ & 1,7 & $(0,3-10,9)$ & 1,4 & $(1,0-2,0)$ \\
\hline \multicolumn{11}{|l|}{ Deserción escolar } \\
\hline No & & & 1,0 & & 1,0 & & 1,0 & & & \\
\hline Sí & & & 1,0 & $(0,3-2,9)$ & 0,9 & $(0,3-2,4)$ & $3,3 * *$ & $(1,6-7,1)$ & & \\
\hline
\end{tabular}

${ }^{*} \mathrm{p}<0,05 ;{ }^{* *} \mathrm{p}<0,01 ;{ }^{* * *} \mathrm{p}<0,001$. Datos Pesados Censo Chile 2002. NSE: número de veces la línea de pobreza urbana.

la actual estructura asistencial de nuestro país (Tabla 5).

La mayoría (66\%) de los niños o adolescentes con un trastorno mental diagnosticado en el estudio no recibió atención de ningún tipo.

La Tabla 6 muestra el nivel de utilización de servicios por grupo diagnóstico. A excepción de los trastornos asociados al consumo de sustancias, los sujetos estudiados recurrieron mayoritariamente a los servicios en el contexto escolar; destacando una mayor proporción relativa de consultantes a servicios especializados en el caso de los trastornos disruptivos.

Finalmente, considerando la proyección al 2010 del Censo 2002, las brechas asistenciales se presentan en la Tabla 7. 
Tabla 4. Comorbilidad de los trastornos psiquiátricos estudiados

\begin{tabular}{|c|c|c|c|c|c|c|}
\hline & $1 \mathrm{di}$ & óstico & 2 dia & sticos & 3 dia & ósticos \\
\hline & OR & $95 \mathrm{Cl}$ & OR & $95 \mathrm{Cl}$ & OR & $95 \mathrm{Cl}$ \\
\hline Género & & & & & & \\
\hline Masculino & 1,0 & & 1,0 & & 1,0 & \\
\hline Femenino & 1,4 & $(1,0-1,9)$ & $1,7^{*}$ & $(1,1-2,8)$ & $4,0^{*}$ & $(1,3-12,9)$ \\
\hline Edad & & & & & & \\
\hline $4-11$ & 1,0 & & 1,0 & & 1,0 & \\
\hline $12-18$ & $0,6^{*}$ & $(0,4-0,9)$ & $0,6^{*}$ & $(0,4-0,9)$ & $2,3^{*}$ & $(1,0-5,3)$ \\
\hline NSE & & & & & & \\
\hline Pobreza $\leq 2$ & 1,0 & & 1,0 & & 1,0 & \\
\hline Pobreza $\leq 5$ & 1,6 & $(0,9-3,0)$ & 1,3 & $(0,6-2,7)$ & 0,6 & $(0,3-1,5)$ \\
\hline Pobreza $\leq 8$ & 1,4 & $(0,7-2,9)$ & 1,0 & $(0,3-2,8)$ & 0,4 & $(0,1-1,3)$ \\
\hline Pobreza $>8$ & 2,0 & $(1,0-3,9)$ & 0,6 & $(0,3-1,3)$ & $0,2^{*}$ & $(0,1-0,7)$ \\
\hline Funcionamiento familiar & $0,7^{*}$ & $(0,5-0,9)$ & $0,3^{* *}$ & $(0,1-0,7)$ & $0,1 * * *$ & $(0,04-0,4)$ \\
\hline Psicopatología familiar & & & & & & \\
\hline No & 1,0 & & 1,0 & & 1,0 & \\
\hline Sí & $2,0^{* * *}$ & $(1,4-2,8)$ & $3,5^{* * *}$ & $(2,0-6,1)$ & $27,0^{* *}$ & $(3,6-201,3)$ \\
\hline Estructura familiar & & & & & & \\
\hline Ambos padres & 1,0 & & 1,0 & & 1,0 & \\
\hline Sólo un padre & $1,7^{* * *}$ & $(1,2-2,5)$ & $3,7^{* \star *}$ & $(2,2-6,1)$ & $3,2^{* *}$ & $(1,4-7,1)$ \\
\hline Otro & 1,1 & $(0,6-2,1)$ & $3,0^{* * *}$ & $(1,6-5,6)$ & $6,0 * * *$ & $(2,6-13,7)$ \\
\hline Deserción escolar & & & & & & \\
\hline No & 1,0 & & 1,0 & & 1,0 & \\
\hline Sí & 1,2 & $(0,6-2,2)$ & 0,7 & $(0,3-1,7)$ & $4,8^{* *}$ & $(1,6-13,9)$ \\
\hline
\end{tabular}

${ }^{*} \mathrm{p}<0,5 ;{ }^{* *} \mathrm{p}<0,01 ;{ }^{* * *} \mathrm{p}<0,001 ;-$ no casos. Datos Pesados Censo Chile 2002. NSE: número de veces la línea de pobreza urbana.

Tabla 5. Uso de servicios por el total de la muestra estudiada $(n=1.558)$

\begin{tabular}{|c|c|c|c|c|c|c|c|c|c|c|c|c|}
\hline & \multicolumn{3}{|c|}{$\begin{array}{c}\text { Sin } \\
\text { diagnóstico } \\
(n=402)\end{array}$} & \multicolumn{3}{|c|}{$\begin{array}{l}\text { Subumbrales } \\
\qquad(n=596)\end{array}$} & \multicolumn{3}{|c|}{$\begin{array}{l}\text { Cualquier } \\
\text { diagnóstico } \\
(n=560)\end{array}$} & \multicolumn{3}{|c|}{$\begin{array}{l}\text { Cualquier } \\
\text { servicio } \\
(n=358)\end{array}$} \\
\hline & $\mathbf{n}$ & $\%$ & SE & $\mathbf{n}$ & $\%$ & SE & $\mathbf{n}$ & $\%$ & SE & $\mathbf{N}$ & $\%$ & SE \\
\hline Servicios en el contexto escolar & 15 & 3,5 & 1,3 & 62 & 10,8 & 2,5 & 104 & 18,1 & 2,9 & 181 & 51,5 & 4,6 \\
\hline Servicios de salud mental ambulatorios & 11 & 8,5 & 1,2 & 55 & 2,1 & 0,9 & 78 & 14,5 & 2,2 & 144 & 40,1 & 3,4 \\
\hline Servicios ambulatorios (Otros) & 5 & 2,2 & 1,0 & 12 & 2,2 & 1,2 & 27 & 4,4 & 1,4 & 44 & 13,0 & 2,6 \\
\hline Otros servicios & 0 & -- & -- & 1 & 0,4 & 0,4 & 5 & 0,5 & 0,3 & 6 & 1,4 & 0,8 \\
\hline Servicios sociales & 6 & 2,3 & 0,9 & 7 & 0,8 & 0,3 & 21 & 3,6 & 1,7 & 34 & 9,6 & 2,7 \\
\hline Cualquier servicio & 33 & 8,1 & 2,0 & 132 & 22,2 & 3,3 & 193 & 33,3 & 3,7 & 358 & 100 & \\
\hline
\end{tabular}

Datos Pesados Censo Chile 2002. 

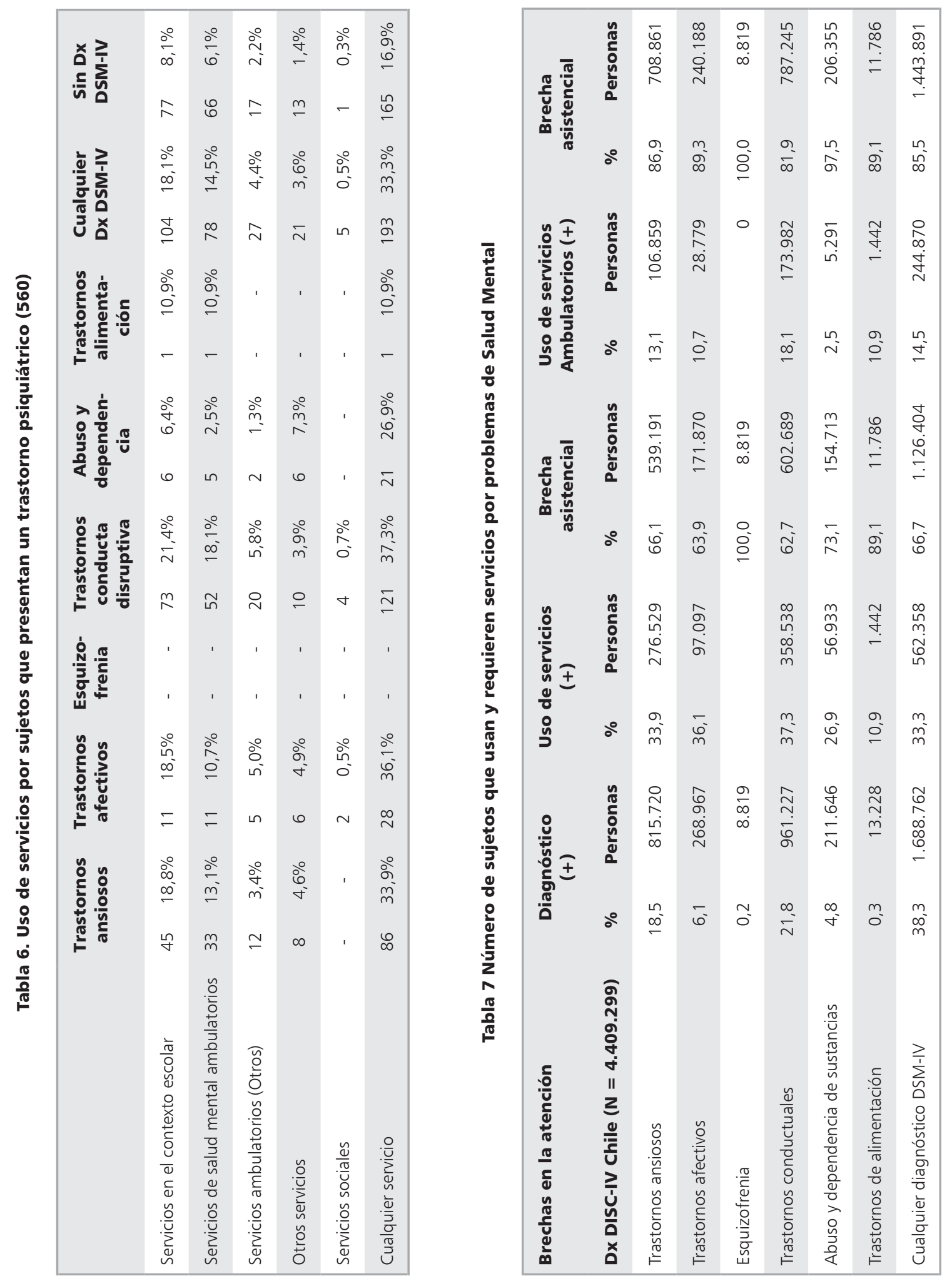
Salud mental infanto-juvenil en Chile - B. Vicente et al

\section{Discusión}

Los resultados demuestran que más de un tercio de la población infanto juvenil en Chile presenta algún trastorno psiquiátrico en un período de 12 meses.

En comparación con otros estudios latinoamericanos las prevalencia nacionales pueden considerarse altas. Tres de los estudios más recientes realizados en Brasil utilizaron como instrumento diagnóstico el Developmental and Well Being Assessment (DAWBA) ${ }^{22}$. El estudio realizado en Tambate entrega una prevalencia general de $2,7 \%{ }^{23}$, el de Ilha de Mare $7 \%{ }^{24}$ y el de Pelotas $10,8 \%{ }^{25}$, lo que contrasta con $22,5 \%$ encontrado en nuestro estudio cuando se utiliza el impedimento D. Dos de los estudios mencionados usaron un diseño en dos etapas que pudo influenciar la selección ${ }^{24,25}$, ya que al incorporar la evaluación clínica las prevalencias tienden a disminuir. Además los grupos etáreos fueron más limitados, 7-14 años en los dos primeros ${ }^{23,24}$ y $11-12$ en el tercero ${ }^{25}$. El estudio nacional presentó prevalencias significativamente más altas en niños que en adolescentes lo que perfila aun más las diferencias aludidas y remite preferentemente a los diseños metodológicos.

Finalmente, considerando estudios aun más recientes nuestra prevalencia con impedimento, resulta notoriamente similar al $22 \%$ obtenido por Merikangas en adolescentes norteamericanos ${ }^{26}$.

Por otro lado, si comparamos nuestros resultados sin considerar impedimento, con el estudio de Puerto Rico ${ }^{15}$, las diferencias son también importantes, ellos obtienen una prevalencia general de $19,8 \%$ comparado con $38,3 \%$ encontrado en Chile, sin embargo, un estudio realizado en México en adolescentes entre 12-17 años ${ }^{27}$ y que usó el Composite Diagnostic Interview (CIDI-A) ${ }^{28}$, obtuvo una prevalencia de $39,4 \%$ lo que es algo mayor a la nacional.

El nivel socioeconómico más alto (Pobreza $>8$ ) es el único que aparece asociado significativamente a la menor presencia de trastornos ansiosos, otros estudios han encontrado asociaciones más fuertes con trastornos externalizantes que internalizantes $^{29,30}$. Sin embargo, estudios recientes muestran resultados más cercanos a los nacionales ${ }^{31-33}$. Estresores relacionados con el ambiente han probado ser parcialmente mediadores entre los problemas de salud mental y el nivel socioeconómico ${ }^{34}$.

Aun cuando no resultan estadísticamente significativas, las mujeres presentan prevalencias mayores que los hombres de trastorno por déficit atencional con hiperactividad. Lo que difiere de lo habitualmente reportado ${ }^{35}$ pero es coincidente con algunos estudios latinoamericanos recientes ${ }^{27,36,37}$. Diferencias que demandan nuevos estudios comparativos.

En general, la presencia de patología se asocia a la disfunción familiar, al antecedente de psicopatología en la familia y no vivir con ambos padres, tanto en el análisis bivariado como multivariado y la comorbilidad es un hallazgo en más de $25 \%$ de los casos.

La mayoría de los niños y adolescentes con diagnóstico no reciben atención y hay una brecha asistencial de $66,6 \%$ para cualquier tipo de trastorno que sube al $85 \%$ cuando se considera sólo el sistema sanitario formal.

La escasa mayor proporción relativa de consultas que en general se advierte en los grupos con diagnóstico, comparado con el grupo sin diagnóstico, requiere avanzar en la identificación de los conceptos de salud y enfermedad de nuestra población junto a las barreras percibidas para acceder a los escasos servicios disponibles, áreas no cubiertas por el presente estudio.

El estudio tiene varias fortalezas:

1) Se uso un instrumento diagnóstico estructurado previamente validado en $\mathrm{Chile}^{17}$.

2) Se obtuvo una muestra nacional representativa usando un diseño estratificado multietápico con una satisfactoria tasa de respuesta.

3) El rango etáreo de los entrevistados fue amplio y se estudiaron otras variables como psicopatología y funcionamiento familiar, nivel socioeconómico y utilización de servicios.

Las limitaciones más importantes son:

1) Se utilizó sólo una fuente de información, los adolescentes mayores de 12 años y los padres o cuidadores en los niños de 4-11 años cuya identificación de psicopatología podría haber estado influenciado por la del informante ${ }^{39-41}$.

2) No se utilizó una medida de discapacidad independiente al DISC-IV lo que ha sido recomendado por algunos autores ${ }^{15}$.

En conclusión podríamos afirmar que las altas prevalencia de trastornos psiquiátricos en la población estudiada evidencia y actualiza un 
problema de salud que no parece estar siendo priorizado adecuadamente por las autoridades, $y$ la consideración de esta información y posterior redistribución de recursos podría impactar positivamente en la reducción de las brechas.

\section{Referencias}

1. Burke KC, Burke JD Jr, Regier DA, Rae DS. Age at onset of selected mental disorders in 5 community populations. Arch Gen Psychiatry 1990; 47 (6): 511-8.

2. Kessler RC, Berglund P, Demler O, Jin R, Merikangas $\mathrm{KR}$, Walters EE. Lifetime prevalence and age of onset distributions of DSM-IV disorders in the National Comorbidity Survey Replication. Arch Gen Psychiatry 2005; 62 (6): 593-602.

3. Rutter M, Sroufe LA. Developmental psychopathology: concepts and challenges. Dev Psychopathol 2000; 12 (3): 265-96.

4. Costello E, Foley DL, Angold A. 10-year research update review: the epidemiology of child and adolescent psychiatric disorders: II. developmental epidemiology. J Am Acad Child Adolesc Psychiatry 2006; 45 (1): 8-25.

5. Leckman JF, Leventhal BL. Editorial: a global perspective on child and adolescent mental health. J Child Psychol Psychiat 2008; 49 (3): 221-5.

6. Patel V, Flischer AJ, Hetrick S, McGorry P. Mental health of young people: a global public health challenge. Lancet 2007; 369 (9569): 1302-13.

7. Kendall PC, Safford S, Flannery-Schroeder E, Webb A. Child anxiety treatment: Outcomes in adolescence and impact on substance use and depression at 7.4-year follow-up. Journal of Consulting and Clinical Psychology 2004; 72: 276-87.

8. Barrett PM, Turner C. Prevention of anxiety symptoms in primary school children: Preliminary results from a universal school-based trial. The British Journal of Clinical Psychology 2001; 40 (Pt 4): 399-410.

9. O'Connell ME, Boat T, Warner KE, eds. Preventing Mental, Emotional, and Behavioral Disorders Among Young People: Progress and Possibilities. The National Academies Press, Washington, DC, 2009; pp 191-220.

10. World Health Organization. Atlas Child and Adolescent Mental Health Resources Global Concerns: Implications for the Future. World Health Organization, Geneva. 2005.

11. Benjet C, Borges G, Medina-Mora ME, Zambrano J, Aguilar-Gaxiola S. Youth mental health in a populous city of the developing world: results from the Mexican
Adolescent Mental Health Survey. J Child Psychol Psychiatry 2009; 50 (4): 386-95.

12. De la Barra F, Toledo V, Rodríguez J. Estudio de salud mental en dos cohortes de niños escolares de Santiago Occidente. IV: desórdenes psiquiátricos, diagnóstico psicosocial y discapacidad. Rev Chil Neuro-Psiquiat 2004; 42 (4): 259-72

13. Vicente B, Kohn R, Rioseco S, Saldivia S, Levav I, Torres S. Lifetime and 12-month Prevalence of DSM-III-R Disorders in the Chile Psychiatric Prevalence Study. Am J Psychiatry 2006; 163 (8): 1362-70.

14. Canino G, Shrout P, Rubio-Stipec M, Bird HR, Bravo M, Ramírez R, et al. The DSM-IV rates of child and adolescent disorders in Puerto Rico: prevalence, correlates, service use, and the effects of impairment. Arch Gen Psychiatry 2004; 61 (1): 85-93.

15. Shaffer D, Fischer P, Lucas CP, Dulcan MK, SchwabStone ME. NIMH Diagnostic Interview Schedule for Children Version IV (NIMH DISC-IV): description, differences from previous versions, and reliability of some common diagnoses. J Am Acad Child Adolesc Psychiatry 2000; 39 (1): 28-38.

16. Saldivia S, Vicente B, Valdivia M, Zúñiga M, Llorente C, Condeza MI. Validación de la entrevista diagnóstica estructurada DISC-IV para la identificación de trastornos psicopatológicos en niños y adolescentes chilenos. Rev Chil Neuro-Psiquiat 2008; 62: 86.

17. Canino G, Shrout PE, Alegria M, Rubio-Stipec M, Chávez LM, Ribera JC, et al. Methodological challenges in assessing children's mental health services utilization. Mental Health Services Research 2002; 4: 97-107.

18. Epstein NB, Baldwin MN, Bishop DS. The McMaster Family Assessment Device. Journal of Martial and Family Therapy 1983; 9: 171-80.

19. Ridenour TA, Daley JG, Reich W. Factor analyses of the family assessment device. Family Process 1999; 38: $497-$ 510.

20. Weissman MM, Wickramarantne P, Adams P, Wolk S, Verdeli $\mathrm{H}$, Olfson M. Brief screening for family psychiatric history: the Family History Screen. Archives of General Psychiatry 2000; 57: 675-82.

21. Herringa $\mathrm{S}$, West $\mathrm{B}$, Berglund $\mathrm{P}$. Applied survey analysis. Boca Raton, Florida: CRC Press. 2010.

22. Goodman R, Ford T, Richards H, Meltzer H, Gatward R. The Development and Well-Being Assessment: description and initial validation of an integrated assessment of child and adolescent psychopathology. Journal of Child Psychology and Psychiatry 2000; 41: 645-57.

23. Fleitlich-Bilyk B, Goodman R. Prevalence of child and adolescent psychiatric disorders in southern Brazil. Journal of the American Academy of Child and Adolescent 
Psychiatry 2004; 43: 727-34.

24. Goodman R, Neves dos Santos D, Robatto Nunes AP, Pereira de Miranda D, Fleitlich-Bilyk B, Almeida Filho N. The Ilha de Maré study: a survey of child mental health problems in a predominantly African-Brazilian rural community. Social Psychiatry and Psychiatric Epidemiology 2005; 40: 11-7.

25. Anselmi L, Fleitlich-Bilyk B, Menezes AM, Araújo CL, Rohde LA. Prevalence of psychiatric disorders in a Brazilian birth cohort of 11-year-olds. Social Psychiatry and Psychiatric Epidemiology 2010; 45: 135-42.

26. Merikangas KR, He JP, Burstein M, Swanson SA, Avenevoli S, Cui L, et al. Lifetime prevalence of mental disorders in U.S. adolescents: results from the National Comorbidity Survey Replication-Adolescent Supplement (NCS-A). Journal of the American Academy of Child and Adolescent Psychiatry 2010; 49: 980-9.

27. Benjet C, Borges G, Medina-Mora ME, Zambrano J, Aguilar-Gaxiola S. Youth mental health in a populous city of the developing world: results from the Mexican Adolescent Mental Health Survey. Journal of Child Psychology and Psychiatry 2009; 50: 386-95.

28. Merikangas K, Avenevoli S, Costello J, Koretz D, Kessler, RC. National comorbidity survey replication adolescent supplement (NCS-A): I. Background and measures. Journal of the American Academy of Child and Adolescent Psychiatry 2009; 48: 367-9.

29. Lipman EL, Offord DR, Boyle MH. What if we could eliminate child poverty? The theoretical effect on child psychosocial morbidity. Social Psychiatry and Psychiatric Epidemiology 1996; 31: 303-7.

30. McLoyd VC. Socioeconomic disadvantage and child development. American Psychologist 1998; 53: 185-204.

31. Carter AS, Wagmiller RJ, Gray SA, McCarthy KJ, Horwitz SM, Briggs-Gowan MJ. Prevalence of DSM-IV disorder in a representative, healthy birth cohort at school entry: sociodemographic risks and social adaptation. Journal of the American Academy of Child and Adolescent Psychiatry 2010 49: 686-98.

32. Roberts RE, Roberts CR, Xing Y. Rates of DSM-IV psychiatric disorders among adolescents in a large metropolitan area. Journal of Psychiatric Research 2007; 41: 959-67.
33. Farbstein I, Mansbach-Kleinfeld I, Levinson D, Goodman R, Levav I, Vograft I, et al. Prevalence and correlates of mental disorders in Israeli adolescents: results from a national mental health survey. Journal of Child Psychology and Psychiatry 2010; 51: 630-9.

34. Amone-P'Olak K, Ormel J, Huisman M, Verhulst FC, Oldehinkel AJ, Burger H. Life stressors as mediators of the relation between socioeconomic position and mental health problems in early adolescence: the TRAILS study. Journal of the American Academy of Child and Adolescent Psychiatry 2009; 48: 1031-8.

35. Polanczyk G, Rohde LA. Epidemiology of attention-deficit/hyperactivity disorder across the lifespan. Current Opinion in Psychiatry 2007; 20: 386-92.

36. Skounti M, Philalithis A, Galanakis E. Variations in prevalence of attention deficit hyperactivity disorder worldwide. European Journal of Pediatrics 2007; 166: 117-23.

37. Rohde LA, Biederman J, Busnello EA, Zimmermann H, Schmitz M, Martins S, et al. ADHD in a school sample of Brazilian adolescents: a study of prevalence, comorbid conditions, and impairments. Journal of the American Academy of Child and Adolescent Psychiatry 1999; 8: 716-22.

38. Montiel-Nava C, Peña JA, López M, Salas M, Zurga JR, Montiel-Barbero I, et al. Estimations of the prevalence of attention deficit hyperactivity disorder in Marabino children. Revista de Neurología 2002; 35: 1019-24.

39. Ford T, Goodman R, Meltzer H. The British child and adolescent mental health survey 1999: The prevalence of DSM-IV disorders. Journal of the American Academy of Child and Adolescent Psychiatry 2003; 42: 1203-11.

40. Bird H, Gould, M, Staghezza B. Aggregating data from multiple informants in child psychiatry epidemiological research. Journal of the American Academy of Child and Adolescent Psychiatry 1992; 31: 78-85.

41. Lahey BB, Flagg EW, Bird HR, Schwab-Stone ME, Canino G, Dulcan MK, et al. The NIMH Methods for the Epidemiology of Child and Adolescent Mental Disorders (MECA) Study: background and methodology. Journal of the American Academy of Child and Adolescent Psychiatry 1996; 35: 855-64. 